CERN-TH/99-402

DF/IST-8.2000

hep-th/0011234

\title{
Supersymmetry of the extreme rotating toroidal black hole
}

\author{
José P. S. Lemosø \\ CENTRA, Departamento de Física, Instituto Superior Técnico, \\ Av. Rovisco Pais 1, 1096 Lisboa, Portugal, \\ \& \\ Theory Divison, CERN, CH-1211, Geneva 23, Switzerland.
}

\begin{abstract}
We study the supersymmetry of the charged rotating toroidal black hole solutions found by Lemos and Zanchin, and show that the only configurations that are supersymmetric are: (i) the non-rotating electrically charged naked singularities already studied by Caldarelli and Klemm, and (ii) an extreme rotating toroidal black hole with zero magnetic and electric charges. For this latter case, the extreme uncharged black hole, we calculate the Killing spinors and show that the configuration preserves the same supersymmetries as the background spacetime.
\end{abstract}

PACS numbers: 04.65.+e; 04.70.-s; 04.60.-m

\footnotetext{
${ }^{1}$ E-mail: lemos@kelvin.ist.utl.pt
} 


\section{Introduction}

There are several motivations to, first, find supersymmetric black hole solutions and, then, analyze their properties. For instance, black holes that are supersymmetric, have zero temperature [1], and in some cases it is possible to associate them with solitons of the theory, interpolating between two distinct homogeneous vacua solutions [2]. In addition, in certain instances, supersymmetric black holes are exact solutions of the theory even when quantum corrections are taken into account [3].

Previous studies looking for supersymmetric configurations appeared in the context of $N=2$ ungauged supergravity whose bosonic sector is pure Einstein-Maxwell theory and for which the black hole solutions, the KerrNewman solutions, are asymptotically flat. It was found that the KerrNewman family is supersymmetric, i.e., admits Killing spinors, when the mass of the solution is equal to the charge. Thus for non-zero angular momentum the supersymmetric solutions are naked singularities, whereas for zero angular momentum, the supersymmetric solution is the extreme ReissnerNordström black hole [4, 5].

Subsequent studies appeared within the context of $N=2$ gauged supergravity, obtained by gauging the symmetry that rotates the fermions (gravitini) of the ungauged theory, in which case the bosonic sector is EinsteinMaxwell with a negative cosmological constant. Due to the presence of the negative cosmological constant there are now three classes of black hole solutions in which the horizons can have spherical, toroidal (cylindrical and planar), and hyperbolical topologies. The supersymmetry of the spherical solutions studied in [6, 7] show that to be supersymmetric the black hole must rotate. Non-rotating supersymmetric configurations are naked singularities. The supersymmetries of toroidal and hyperbolic topologies were studied in a comprehensive paper by Caldarelli and Klemm [8]. For toroidal black holes a result similar to spherical black holes was obtained, while for hyperbolical black holes there are also non-rotating configurations, with magnetic charge, which are supersymmetric [8]. The study of supersymmetries were extended in [9] to include Taub-NUT spacetimes, all of these solutions belonging to the Plebanski-Demianski family of solutions.

A different rotating toroidal (or cylindrical) black hole solution was found in [10] and then generalized to include charge [11], (see also [12], [13] for a review, and the charged solution in the non-rotating case is also discussed 
in [14]). These solutions are not isometric to the toroidal solutions found by Klemm and Vanzo [15, which are a special case of the Plebanski-Demianski metrics, and whose supersymmetries were studied in [8]. The main aim of this paper is to study the supersymmetries of the toroidal (or cylindrical) rotating charged black holes in anti-de Sitter spacetime found in 10, 11. We study the integrability conditions, and find that the supersymmetric black hole has zero charge, must rotate with extreme angular velocity and thus has zero temperature. The corresponding Killing spinor is explicitly displayed and it is shown that the extreme black hole preserves the same number of supersymmetries as the original anti-de Sitter spacetime. There are other non-rotating solutions, representing charged naked singularities, which are supersymmetric [8].

\section{2. $N=2$ gauged supergravity}

In $N=2$ supergravity there are four bosonic degrees of freedom, represented by a graviton $e_{m}^{a}$ and a Maxwell gauge field $A_{m}$, and four fermionic degrees of freedom, represented by two real gravitini $\psi_{m}^{i}(i=1,2)$, usually combined into a single complex spinor $\psi_{m}=\psi_{m}^{1}+i \psi_{m}^{2}$. Gauged $N=2 \mathrm{su}-$ pergravity can be obtained from the ungauged theory by gauging the $\mathrm{SO}(2)$ symmetry that rotates the gravitini, with the corresponding appearance of a minimal gauge coupling $\alpha$ between the photons and the gravitini. Invariance of the action under local supersymmetric transformations requires a mass term for the gravitini and the introduction of a negative cosmological constant.

The action is

$$
\begin{aligned}
S= & \int d^{4} x\left\{R+6 \alpha^{2}-F_{m n} F^{m n}-2 \bar{\psi}_{m} \gamma^{m n p} D_{n} \psi_{p}+2 \alpha \bar{\psi}_{m} \gamma^{m n} \psi_{n}\right. \\
& \left.-\frac{i}{2}\left(F^{m n}+\hat{F}^{m n}\right) \bar{\psi}_{p} \gamma_{[m} \gamma^{p q} \gamma_{n]} \psi_{q}\right\} .
\end{aligned}
$$

We use the same notation as in $[8]: a, b, \ldots$ are tangent space indices, and $m, n, \ldots$ are world indices. The signature is $(-,+,+,+)$, and the real representation of the gamma matrices $\gamma_{a}$ is used. The $\gamma$ matrices satisfy $\left\{\gamma_{a}, \gamma_{b}\right\}=$ $2 \eta_{a b}$, antisymetrization is defined by $\gamma_{a b} \equiv \gamma_{[a} \gamma_{b]} \equiv \frac{1}{2}\left[\gamma_{a}, \gamma_{b}\right]$, and $\gamma_{5}=\gamma_{0123}=$ $\gamma_{0} \gamma_{1} \gamma_{2} \gamma_{3}$. From equation (1) one sees that the cosmological constant $\Lambda$ is related to the gauge coupling $\alpha$ by $\Lambda=-3 \alpha^{2} . D_{m}$ is the gauge covariant 
derivative given by

$$
D_{m}=\nabla_{m}-i \alpha A_{m}
$$

with $\nabla_{m}$ being the Lorentz covariant derivative

$$
\nabla_{m}=\partial_{m}+\frac{1}{4} \omega_{m}^{a b} \gamma_{a b}
$$

The spin connection is given in terms of the tetrads and spinors by

$$
\begin{aligned}
\omega_{m a b}= & e_{a}^{n}\left\{\partial_{[m} e_{b n]}-\frac{1}{2} \operatorname{Re}\left(\bar{\psi}_{m} \gamma_{b} \psi_{n}\right)\right\} \\
& -e_{b}^{n}\left\{\partial_{[m} e_{a n]}-\frac{1}{2} \operatorname{Re}\left(\bar{\psi}_{m} \gamma_{a} \psi_{n}\right)\right\} \\
& -e_{a}^{l} e_{b}^{n} e_{m}^{c}\left\{\partial_{[l} e_{c n]}-\frac{1}{2} \operatorname{Re}\left(\bar{\psi}_{l} \gamma_{c} \psi_{n}\right)\right\} .
\end{aligned}
$$

$F_{m n}$ and $\hat{F}_{m n}$ denote the standard and the supercovariant field strength, respectively, with

$$
\hat{F}_{m n}=F_{m n}-\operatorname{Im}\left(\bar{\psi}_{m} \psi_{n}\right) .
$$

The action (11) is invariant under the usual general coordinate, local Lorentz, and $\mathrm{U}(1)$ gauge transformation. It is also invariant under the following local $N=2$ supersymmetric transformations

$$
\delta e_{m}^{a}=\operatorname{Re}\left(\bar{\epsilon} \gamma^{a} \psi_{m}\right), \quad \delta A_{m}=\operatorname{Im}\left(\bar{\epsilon} \psi_{m}\right), \quad \delta \psi_{m}=\hat{\nabla}_{m} \epsilon
$$

where $\hat{\nabla}_{m}$ is the supercovariant derivative defined by

$$
\hat{\nabla}_{m}=D_{m}+\frac{1}{2 \ell} \gamma_{m}+\frac{i}{4} \hat{F}_{a b} \gamma^{a b} \gamma_{m}
$$

and $\epsilon$ is an infinitesimal Dirac spinor.

To study a theory one should look for solutions of the equations of motion. One way to find solutions of action (四) is to consider its bosonic sector alone, by putting the fermionic fields equal to zero, $\psi_{m}=0$, and then find solutions within this sector. A solution of the bosonic sector is also a solution of the full supergravity theory if the solution admits a Dirac Killing spinor $\epsilon$ whose supercovariant derivative is zero, $\hat{\nabla}_{m} \epsilon=0$. Indeed, in this case, the last equation of (6) defines a Killing spinor equation for the Killing spinor $\epsilon$, and tells that the fermionic fields remain identically zero for any supersymmetric 
transformation. The corresponding bosonic solution is then a true solution of the theory.

Thus, among the solutions of the bosonic sector one looks for those which are invariant under supersymmetric transformations, i.e., solutions which admit a Killing spinor obeying the equation

$$
\hat{\nabla}_{m} \epsilon=0 \text {. }
$$

The integrability conditions for (8) are then

$$
\hat{R}_{m n} \epsilon \equiv\left[\hat{\nabla}_{m}, \hat{\nabla}_{n}\right] \epsilon=0,
$$

where $\hat{R}_{m n}$ is the supercurvature. Note that since (9) is a local equation, it gives a necessary condition for the existence of Killing spinors, but not a sufficient one. In order to assure that there are Killing spinors one has to integrate equation (8). In the following sections, we shall solve (8) and (9) for the black hole spacetimes introduced in [10, 11].

\section{The toroidal solutions in general relativity}

By putting to zero the fermionic sector of $N=2$ supergravity in (1) one obtains the Einstein-Maxwell action

$$
S=\int d^{4} x\left(R+6 \alpha^{2}-F_{m n} F^{m n}\right) .
$$

A rotating solution, which can represent a black hole, may be found from the equations of motion following from (10) (see [11]). The rotating solution can be obtained from the non-rotating toroidal metric by mixing time and angle into a new time and a new angle. Since angles are periodic, this is not a proper coordinate transformation, yielding in this way a new solution globally different from the static one (see [10]). This metric is thus different from the rotating toroidal metric found by Klemm and Vanzo [15] which cannot be obtained by this forbidden coordinate mixing, is instead obtainable from the general Petrov type- $D$ solution. Our charged rotating toroidal solution is given by

$$
\begin{aligned}
d s^{2}= & -\frac{\Delta}{(\alpha r)^{2}}(d t-\lambda d \phi)^{2}+\frac{(\alpha r)^{2}}{\Delta} d r^{2}+ \\
& +(\alpha r)^{2} d z^{2}+r^{2}\left(\alpha^{2} \lambda d t-d \phi\right)^{2},
\end{aligned}
$$




$$
A=-\frac{2 Q_{E}}{\alpha r}(d t-\lambda d \phi)-2 Q_{M} z\left(d \phi-\lambda \alpha^{2} d t\right) .
$$

The Ricci curvature $R_{m n}$ can be found from (11), which we will do later. The Maxwell tensor $F_{m n}$ can be found from the Maxwell curvature 2-form $F=F_{m n} d x^{m} \wedge d x^{n}$. From (12) it is given by

$$
F=d A=\frac{2 Q_{E}}{\alpha r^{2}} d r \wedge(d t-\lambda d \phi)-2 Q_{M} d z \wedge\left(d \phi-\lambda \alpha^{2} d t\right) .
$$

In this solution, the ranges of the time and radial coordinates are $-\infty<t<$ $+\infty, 0 \leq r<+\infty$. The topology of the two dimensional space, $t=$ constant and $r=$ constant, generated by the group $G_{2}$ can be (i) $R \times S^{1}$, the standard cylindrical symmetric model, with orbits diffeomorphic either to cylinders or to $R$ (i.e, $G_{2}=R \times U(1)$ ), with $-\infty<z<+\infty, 0 \leq \phi<2 \pi$, (ii) $S^{1} \times S^{1}$ the flat torus $T^{2}$ model $\left(G_{2}=U(1) \times U(1)\right)$ with $0 \leq \alpha z<2 \pi, 0 \leq \phi<2 \pi$, and (iii) $R^{2}$, the planar model with $-\infty<z<+\infty,-\infty<(\phi / \alpha)<+\infty$, but in this case the black hole (in fact, a black membrane) does not rotate.

In order to proceed, we choose the following tetrads

$$
e^{0}=\frac{\sqrt{\Delta}}{\alpha r}(d t-\lambda d \phi), \quad e^{1}=\frac{\alpha r}{\sqrt{\Delta}} d z, \quad e^{2}=\alpha r d z, \quad e^{3}=r\left(\lambda \alpha^{2} d t-d \phi\right) .
$$

Then equations (11)-(12) can be written as

$$
\begin{gathered}
d s^{2}=-\left(e^{0}\right)^{2}+\left(e^{1}\right)^{2}+\left(e^{2}\right)^{2}+\left(e^{3}\right)^{2}, \\
A=-\frac{2 Q_{E}}{\Delta} e^{0}+2 Q_{M} \frac{z}{r} e^{3} .
\end{gathered}
$$

The Maxwell 2-form is

$$
F=-\frac{2 Q_{E}}{\alpha r^{2}} e^{0} \wedge e^{1}+\frac{2 Q_{M}}{\alpha r^{2}} e^{2} \wedge e^{3} .
$$

The functions and parameters appearing in (11)-(17) are: $\Delta=4 c^{2}-b \alpha r+$ $\alpha^{4} r^{4}, \lambda=\frac{1}{\alpha} \frac{\sqrt{1-\sqrt{1-\frac{8 J^{2} \alpha^{2}}{9 M^{2}}}}}{\sqrt{1+\sqrt{1-\frac{8 J^{2} \alpha^{2}}{9 M^{2}}}}}$ a specific angular momentum parameter, $J$ and $M$ being the angular momentum and mass per unit length of the system, respectively, $c^{2}=4 Q^{2}\left(1-\lambda^{2} \alpha^{2}\right)$ with $Q^{2}=Q_{E}^{2}+Q_{M}^{2}, Q_{E}$ and $Q_{M}$ being 
the electric and magnetic charges per unit length, respectively, and $b=$ $4 M \frac{1-\lambda^{2} \alpha^{2}}{1+\frac{1}{2} \lambda^{2} \alpha^{2}}$. Note that $M=0$ implies $J=0$. In [11] another specific angular momentum parameter $a$ was used, related to $\lambda$ by $\lambda=a / \sqrt{1-\frac{1}{2} a^{2} \alpha^{2}}$. For the toroidal case, the mass $\bar{M}$, angular momentum $\bar{J}$, and charge $\bar{Q}$ are given in terms of $M, J$ and $Q$ by $\bar{M}=\frac{2 \pi}{\alpha} M, \bar{J}=\frac{2 \pi}{\alpha} J$, and $\bar{Q}=\frac{2 \pi}{\alpha} Q$ [11]. When $\Delta$ has two roots the solution represents a black string (in the cylindrical model) or a black hole (in the toroidal model), when it has one root it represents, correspondingly, an extreme black string or an extreme black hole, and when it has no roots it represents, correspondingly, a singular naked straight line or a singular closed line.

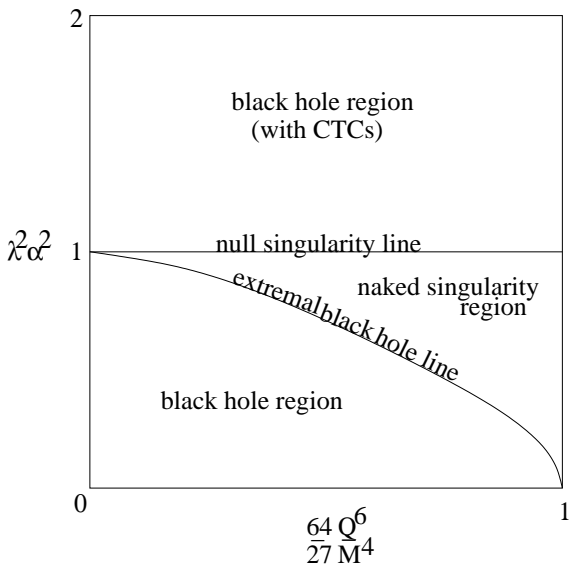

Figure 1. The five regions and lines which yield solutions of different nature are shown in the parameter space $\lambda \times Q$ in appropriate units of $\alpha$ and mass, respectively.

In figure 1, we show the black hole and naked singularity regions, and the extremal black hole line dividing those two regions, as well as the other solutions in the upper part of the figure. The regions are

(i) $0 \leq \lambda^{2} \alpha^{2}<1-\frac{64 Q^{6}}{27 M^{4}}\left(1+\alpha^{2} \lambda^{2}\right)^{4}$

Black hole region

(ii) $\lambda^{2} \alpha^{2}=1-\frac{64 Q^{6}}{27 M^{4}}\left(1+\alpha^{2} \lambda^{2}\right)^{4}$

Extreme black hole line

(iii) $1-\frac{64 Q^{6}}{27 M^{4}}\left(1+\alpha^{2} \lambda^{2}\right)^{4}<\lambda^{2} \alpha^{2}<1$

Naked singularity region

(iv) $\lambda^{2} \alpha^{2}=1$

Null singularity line 
(v) $1<\lambda^{2} \alpha^{2} \leq 2$

Pathological black hole region

(black holes with closed timelike curves)

For $J>\sqrt{\frac{9 M}{3 \alpha}}$, the specific angular momentum parameter $\lambda$ turns complex, and the metric is ill-defined. The extreme case is given when $Q$ is connected to $\lambda$ through the relation $Q^{6}=\frac{27}{64} \frac{1-\lambda^{2} \alpha^{2}}{1+\frac{1}{2} \lambda^{2} \alpha^{2}}$. For the Penrose diagrams see [11, 16].

In the uncharged case, one has three regions only [10,

(i) $0 \leq \lambda^{2} \alpha^{2}<1$

Black hole region

(ii) $\lambda^{2} \alpha^{2}=1$

Extreme black hole line

(iii) $1<\lambda^{2} \alpha^{2} \leq 2$

Naked singularity region

For the Penrose diagrams of the uncharged case, see [12].

The black hole as well as the naked singularity solutions can appear through gravitational collapse [17, 18, 19] and the non-rotating configurations have thermodynamical properties related to Reissner-Nordström black holes [20, 21, 22, 23].

\section{The integrability conditions and Killing spinors for the charged configurations}

In order to find the full covariant derivative we need to compute the spin connections for the tetrad (14) using equation (4) with the spinor part set to zero (or, alternatively, solving the first Cartan equation $d e^{a}+\omega^{a}{ }_{b} \wedge e^{b}=0$ ).

The non-zero spin connections are:

$$
\begin{gathered}
\omega_{t}^{01}=\frac{1}{2} \frac{1}{\alpha^{2} r^{2}}\left(\Delta^{\prime}-2 \frac{\Delta}{r}\right), \\
\omega_{\phi}^{01}=-\frac{1}{2} \frac{\lambda}{\alpha^{2} r^{2}}\left(\Delta^{\prime}-2 \frac{\Delta}{r}\right), \\
\omega_{z}^{12}=-\frac{1}{2} \frac{\sqrt{\Delta}}{r}, \\
\omega_{t}^{13}=-\alpha \lambda \frac{\Delta}{r},
\end{gathered}
$$




$$
\omega_{\phi}^{13}=\frac{\sqrt{\Delta}}{\alpha r}
$$

with $^{\prime} \equiv d / d r$. Then the supercovariant derivatives are

$$
\begin{aligned}
\hat{\nabla}_{t}= & \partial_{t}-i \alpha\left(-\frac{2 Q_{E}}{\alpha r}+2 Q_{M} \lambda \alpha^{2} z\right) \\
& +\left(\frac{1}{2} \alpha+\frac{i}{4} F_{a b} \gamma^{a b}\right)\left(\frac{\sqrt{\Delta}}{\alpha r} \gamma_{0}+r \lambda \alpha^{2} \gamma_{3}\right) \\
& +\frac{1}{4} \frac{1}{\alpha^{2} r^{2}}\left(\Delta^{\prime}-2 \frac{\Delta}{r}\right) \gamma_{0} \gamma_{1}-\frac{1}{2} \alpha \lambda \frac{\Delta}{r} \gamma_{1} \gamma_{3}, \\
\hat{\nabla}_{r}= & \partial_{r}+\frac{1}{2} \frac{\alpha^{2} r}{\sqrt{\Delta}} \gamma_{1}+\frac{\alpha r}{\sqrt{\Delta}} \frac{i}{4} F_{a b} \gamma^{a b} \gamma_{1}, \\
\hat{\nabla}_{z}= & \partial_{z}+\frac{1}{2} \alpha^{2} r \gamma_{2}+\alpha r \frac{i}{4} F_{a b} \gamma^{a b} \gamma_{2}-\frac{1}{4} \frac{\sqrt{\Delta}}{r} \gamma_{1} \gamma_{2}, \\
\hat{\nabla}_{\phi}= & \partial_{\phi}-i \alpha\left(\frac{2 Q_{E} \lambda}{\alpha r}-2 Q_{M} z\right) \\
& -\left(\frac{1}{2} \alpha+\frac{i}{4} F_{a b} \gamma^{a b}\right)\left(\frac{\lambda \sqrt{\Delta}}{\alpha r} \gamma_{0}+r \gamma_{3}\right) \\
& -\frac{1}{4} \frac{\lambda}{\alpha^{2} r^{2}}\left(\Delta^{\prime}-2 \frac{\Delta}{r}\right) \gamma_{0} \gamma_{1}+\frac{1}{2} \frac{\sqrt{\Delta}}{\alpha r} \gamma_{1} \gamma_{3},
\end{aligned}
$$

with $(i / 4) F_{a b} \gamma^{a b}=\left(i / \alpha r^{2}\right)\left(Q_{E} \gamma_{0} \gamma_{1}+Q_{M} \gamma_{2} \gamma_{3}\right)$.

Like in the case of Reissner-Nordström-anti de Sitter [6], of Kerr-Newmananti-de Sitter [8], and for the other class of rotating topological black holes in anti-de Sitter spacetimes [8] one finds that the super-Riemann tensor (9) can be written as a product

$$
\hat{R}_{m n}=\mathcal{P} \mathcal{G}_{m n} \mathcal{O}
$$

where $\mathcal{P}$ is a projection operator given by

$$
\mathcal{P}=\frac{\alpha r^{2}}{2 Q} i F_{a b} \gamma^{a b} \gamma_{1}=\frac{\alpha r^{2}}{2 Q} i\left(-F_{01} \gamma_{0}+F_{23} \gamma_{1} \gamma_{2} \gamma_{3}\right)
$$

$\mathcal{G}_{m n}$ is $\gamma_{m n}$ times some function of $r$, and $\mathcal{O}$ is given by

$$
\mathcal{O}=\frac{\sqrt{\Delta}}{\alpha r}+\alpha r \gamma_{1}+\sigma \mathcal{P}
$$


where $\sigma \equiv\left[\frac{b}{4 Q}-\frac{2 Q}{\alpha r}\left(1-\tilde{\lambda}^{2}\right)\right]$, and we have put $\tilde{\lambda} \equiv \lambda \alpha$, to simplify the notation. In the real representation of the gamma matrices we find

$$
\mathcal{O}=\left(\begin{array}{cccc}
\frac{\sqrt{\Delta}}{\alpha r}-\alpha r & 0 & i \frac{Q_{M}}{Q} \sigma & -i \frac{Q_{E}}{Q} \sigma \\
0 & \frac{\sqrt{\Delta}}{\alpha r}+\alpha r & i \frac{Q_{E}}{Q} \sigma & i \frac{Q_{M}}{Q} \sigma \\
-i \frac{Q_{M}}{Q} \sigma & -i \frac{Q_{E}}{Q} \sigma & \frac{\sqrt{\Delta}}{\alpha r}-\alpha r & 0 \\
i \frac{Q_{E}}{Q} \sigma & -i \frac{Q_{M}}{Q} \sigma & 0 & \frac{\sqrt{\Delta}}{\alpha r}+\alpha r
\end{array}\right) .
$$

For $Q \neq 0, \mathcal{G}_{m n}$ and $\mathcal{P}$ are non-singular and the integrability conditions for the existence of Killing spinors are equivalent to the vanishing of the determinant of $\mathcal{O}$. One then finds

$$
\begin{gathered}
\operatorname{det} \mathcal{O}=\left[\left(\frac{\Delta}{\alpha^{2} r^{2}}-\alpha^{2} r^{2}\right)-\sigma^{2}\right]^{2}-4 \frac{Q_{M}^{2}}{Q^{2}} \sigma^{2} \alpha^{2} r^{2}= \\
{\left[\frac{4 Q^{2}}{\alpha^{2} r^{2}} \tilde{\lambda}^{2}\left(1-\tilde{\lambda}^{2}\right)-\frac{b}{\alpha r} \tilde{\lambda}^{2}-\frac{b^{2}}{4 Q^{2}}\right]^{2}-\left[\frac{2 Q_{M} b \alpha r}{4 Q^{2}}-4 Q_{M}\left(1-\tilde{\lambda}^{2}\right)\right]^{2}}
\end{gathered}
$$

Since $Q \neq 0$ the constraint implies either $\tilde{\lambda}=0$ (i.e., $J=0$ ), the nonrotating case, or $\tilde{\lambda}=1$, the null singularity rotating case, $(\tilde{\lambda}$ can also take negative values, in this case $\tilde{\lambda}=-1$, but the results do not differ, and we stick to the positive value of $\tilde{\lambda}$ always). All these cases yield charged naked singularities, which we analyze now.

(i) $\tilde{\lambda}=0$ : For no-rotation the determinant (31) simplifies to

$$
\begin{array}{r}
\operatorname{det} \mathcal{O}=\frac{1}{(4 Q)^{4}} \quad\left[b^{2}-\left(2 Q_{M} b \alpha r-16 Q_{M} Q^{2}\right)\right] \times \\
{\left[b^{2}+\left(2 Q_{M} b \alpha r-16 Q_{M} Q^{2}\right)\right] .}
\end{array}
$$

Then $\operatorname{det} \mathcal{O}=0$ implies

$$
\begin{gathered}
Q_{M} b \alpha=0, \\
b^{2}= \pm 16 Q_{M} Q^{2} .
\end{gathered}
$$

As stated in [9], equation (33) is a constraint, while (34) is the saturated Bogomol'nyi bound for the configurations. For $\alpha \neq 0$ (which we usually assume, see [4, 5, \&] for $\alpha=0$ ), one finds $Q_{M}=0 \Rightarrow b=0$, i.e., $M=0$. Then $\Delta=4 Q_{E}^{2}+\alpha^{4} r^{4}$. This is a massless charged naked singularity with no-rotation, in the nomenclature of Romans [6] called a cosmic electric monopole. Caldarelli and Klemm [8] found this supersymmetric 
solution and have displayed the corresponding Killing spinors. Note, however, that when treating the toroidal case one has to change $b, Q_{M}$, and $Q$ defined for the cylindrical model as charges per unit length, to the bar quantities $\bar{b}, \bar{Q}_{M}$, and $\bar{Q}$, defined previously for the toroidal model. Then, since (34) is not homogeneous in those three charges, it turns into $\bar{b}^{2}= \pm 16 \alpha \bar{Q}_{M} \bar{Q}^{2}$, where a new factor $\alpha$ has appeared, which is the integrability condition found in [8] (see also [9]). In [8] was then found another supersymmetric configuration with $\alpha=0$, and $\bar{M}=0$.

(ii) $\tilde{\lambda}=1$ : The charged configuration is a rotating null singularity. The determinant (31) is now

$$
\operatorname{det} \mathcal{O}=\left[\frac{b}{\alpha r} \tilde{\lambda}^{2}+\frac{b^{2}}{4 Q^{2}}\right]^{2}-\left[\frac{2 Q_{M} b \alpha r}{4 Q^{2}}-4 Q_{M}\left(1-\tilde{\lambda}^{2}\right)\right]^{2}
$$

However, since $\tilde{\lambda}=1$, then automatically $b=0$, and (35) is trivially satisfied. In this case $\Delta=\alpha^{2} r^{2}$ and the integrability condition $\mathcal{O} \epsilon=0$ simplifies to

$$
\mathcal{O} \epsilon=(2 \alpha r)\left[\frac{1}{2}\left(1+\gamma_{1}\right)\right] \epsilon=0,
$$

where $\frac{1}{2}\left(1+\gamma_{1}\right)$ is a projection operator. To find whether the solution is supersymmetric or not one should construct the Killing spinors. Using equations (23)-(26) and (36) we find

$$
\begin{gathered}
\partial_{t} \epsilon+\left[i \frac{Q_{E}}{r}\left(1+\gamma_{0} \gamma_{3}\right)-i 2 Q_{M} \alpha^{2} z+i \frac{Q_{M}}{r}\left(1-\gamma_{0} \gamma_{3}\right)\right] \epsilon=0 \\
\partial_{r} \epsilon+\left[\frac{1}{2 r} \gamma_{1}+\frac{i}{\alpha^{2} r^{3}}\left(Q_{E} \gamma_{0}-Q_{M} \gamma_{1} \gamma_{2} \gamma_{2}\right)\right] \epsilon=0 \\
\partial_{z} \epsilon+\frac{i}{r}\left(Q_{E} \gamma_{0} \gamma_{2}-Q_{M} \gamma_{3}\right) \epsilon=0 \\
\partial_{\phi} \epsilon-\left[i \frac{Q_{E}}{r}\left(1+\gamma_{0} \gamma_{3}\right)-i 2 Q_{M} \alpha^{2} z+i \frac{Q_{M}}{r}\left(1-\gamma_{0} \gamma_{3}\right)\right] \epsilon=0
\end{gathered}
$$

Using the techniques of Romans [6] (see also [8]) we find that there are no Killing spinor solutions to equations (37)-(40). Thus the only supersymmetric charged toroidal configurations are the static ones studied in $[8]$. We now turn to the uncharged case. 


\section{The Killing spinors for the extreme rotating uncharged black hole spacetime}

When $Q=0$ (i.e., $Q_{E}=Q_{M}=0$ ) the integrability conditions (9) yield

$$
\hat{R}_{m n}=\mathcal{G}_{m n} b=0,
$$

where $\mathcal{G}_{m n}$ is some function of $r$ times $\gamma_{m} \gamma_{n}$. Thus $b=0$ which, since $b=4 M \frac{1-\tilde{\lambda}^{2}}{1+\frac{1}{2} \hat{\lambda}^{2}}$, yields 2 cases: (i) $M=0$, the anti-de Sitter space with cylindrical or toroidal topology, and (ii) $\tilde{\lambda}=1$, the extreme rotating toroidal black hole [10]. We now analyze each case.

(i) $M=0$ : The Killing spinor equations (23)-(26) reduce to

$$
\begin{aligned}
\hat{\nabla}_{t} \epsilon & =\left[\partial_{t}+\frac{1}{2} \alpha^{2} r\left(\gamma_{0}+\gamma_{0} \gamma_{1}\right)\right] \epsilon=0, \\
\hat{\nabla}_{r} \epsilon & =\left[\partial_{r}+\frac{1}{2 r} \gamma_{1}\right] \epsilon=0, \\
\hat{\nabla}_{z} \epsilon & =\left[\partial_{z}+\frac{1}{2} \alpha^{2} r\left(\gamma_{2}-\gamma_{1} \gamma_{2}\right)\right] \epsilon=0, \\
\hat{\nabla}_{\phi} \epsilon & =\left[\partial_{\phi}-\frac{1}{2} \alpha r\left(\gamma_{3}-\gamma_{1} \gamma_{3}\right)\right] \epsilon=0 .
\end{aligned}
$$

Solving this set of equations yields

$$
\begin{aligned}
\epsilon= & {\left[\sqrt{r} \frac{1}{2}\left(1-\gamma_{1}\right)+\frac{1}{\sqrt{r}} \frac{1}{2}\left(1+\gamma_{1}\right)\right]\left[1-\gamma_{0}\left(1+\gamma_{1}\right) \frac{t}{2}\right] } \\
& {\left[1-\gamma_{2}\left(1+\gamma_{1}\right) \frac{z}{2}\right]\left[1+\gamma_{3}\left(1+\gamma_{1}\right) \frac{\phi}{2}\right] \epsilon_{0}, }
\end{aligned}
$$

where $\epsilon_{0}$ is a constant Dirac spinor

$$
\epsilon_{0}=\left(\begin{array}{l}
a \\
b \\
c \\
d
\end{array}\right) .
$$

In order that $\epsilon_{0}$ is independent of the angle $\phi$ one has $\frac{1}{2}\left(1+\gamma_{1}\right) \epsilon_{0}=0$, which amounts to say that $b=d=0$. Thus

$$
\epsilon=\sqrt{r}\left[\frac{1}{2}\left(1-\gamma_{1}\right)\right] \epsilon_{0},
$$

defining two linearly independent Dirac spinors (see also [8]). 
(ii) $\tilde{\lambda}=1$ : This is the extreme black hole [10, 11]. Using the integrability conditions (i.e., $\mathcal{O} \epsilon=0)$ in equations $(23)-(26)$ one gets

$$
\begin{aligned}
\hat{\nabla}_{t} \epsilon & =\left[\partial_{t}+\frac{1}{2} \alpha^{2} r\left(\gamma_{0}+\gamma_{3}+\gamma_{0} \gamma_{1}-\gamma_{1} \gamma_{3}\right)\right] \epsilon=0, \\
\hat{\nabla}_{r} \epsilon & =\left[\partial_{r}+\frac{1}{2 r} \gamma_{1}\right] \epsilon=0, \\
\hat{\nabla}_{z} \epsilon & =\left[\partial_{z}+\frac{1}{2} \alpha^{2} r\left(\gamma_{2}-\gamma_{1} \gamma_{2}\right)\right] \epsilon=0, \\
\hat{\nabla}_{\phi} & =\left[\partial_{\phi}-\frac{1}{2} \alpha r\left(\gamma_{0}+\gamma_{3}+\gamma_{0} \gamma_{1}-\gamma_{1} \gamma_{3}\right)\right] \epsilon=0 .
\end{aligned}
$$

Solving this set of equations yields

$$
\begin{aligned}
\epsilon= & {\left[\sqrt{r} \frac{1}{2}\left(1-\gamma_{1}\right)+\frac{1}{\sqrt{r}} \frac{1}{2}\left(1+\gamma_{1}\right)\right]\left[1-\left(\gamma_{0}+\gamma_{3}\right)\left(1+\gamma_{1}\right) \frac{t}{2}\right] } \\
& {\left[1-\gamma_{2}\left(1+\gamma_{1}\right) \frac{z}{2}\right]\left[1+\left(\gamma_{0}+\gamma_{3}\right)\left(1+\gamma_{1}\right) \frac{\phi}{2}\right] \epsilon_{0}, }
\end{aligned}
$$

where, again, $\epsilon_{0}$ is a constant Dirac spinor

$$
\epsilon_{0}=\left(\begin{array}{l}
a \\
b \\
c \\
d
\end{array}\right) .
$$

In order that $\epsilon_{0}$ is independent of the angle $\phi$ one has $\frac{1}{2}\left(1+\gamma_{1}\right) \epsilon_{0}=0$, which amounts to say that $b=d=0$. Thus,

$$
\epsilon=\sqrt{r}\left[\frac{1}{2}\left(1-\gamma_{1}\right)\right] \epsilon_{0},
$$

defining, again, two linearly independent Dirac spinors. The extreme uncharged black hole has two supersymmetries, the same number as the background spacetime, and in these coordinates the Killing spinors have the same expression.

It is important to show that the extreme black hole has zero temperature. Indeed [10,

$$
T=\frac{\alpha}{2 \pi} \frac{3}{2} M^{1 / 3}\left[\frac{1-\tilde{\lambda}^{2}}{2\left(1+\frac{1}{2} \tilde{\lambda}^{2}\right)}\right]^{1 / 3}\left(1-\tilde{\lambda}^{2}\right)^{1 / 2} .
$$


For the extreme supersymmetric black hole, $\tilde{\lambda}=1$, yielding a black hole of zero temperature.

\section{Conclusions}

We found that the uncharged toroidal (or cylindrical) black hole must rotate extremally to be supersymmetric. There are no breaks of supersymmetry: there are two supersymmetries, as in the original spacetime. This black hole has zero temperature. The uncharged black hole with planar topology cannot be supersymmetric since it does not rotate. In this sense, the topology of the spacetime plays a role in the supersymmetry analysis.

For charged configurations we found that the supersymmetric ones are static naked singularities with zero magnetic charge. There are no rotating supersymmetric charged configurations.

\section{Acknowledgments}

I thank Observatório Nacional-MCT do Rio de Janeiro for hospitality while this work was being initiated. I thank the Theory Division - CERN, where the most part of this work has been done, for hospitality and for providing an Associate grant for three months. I also thank Vilson Zanchin for conversations, and FAPERGS for a grant to stay at Universidade Federal de Santa Maria, Rio Grande do Sul, where this work was finished.

\section{References}

[1] G. W. Gibbons, in "Unified Theories of Elementary Particles", eds. P. Breitenhlohner, H. P. Dürr (Springer-Verlag, Berlim 1982).

[2] G. W. Gibbons, in "Supersymmetry, Supergravity and Related Topics", eds. F. Aguila, J. A. Azcárraga, L. E. Ibañez (World Scientific, Singapore 1985).

[3] R. Kallosh, Phys. Lett. B282 (1992) 80.

[4] G. W. Gibbons, C. M. Hull, Phys. Lett. B109 (1982) 190. 
[5] K. T. Todd, Phys. Lett. B121 (1983) 241.

[6] L. J. Romans, Nucl. Phys.B 383, 395 (1992).

[7] V. A. Kostelecký, M. J. Perry, Phys. Lett. B 371, 191 (1996).

[8] M. M. Caldarelli, D. Klemm, Nucl. Phys. B545 (1999) 434.

[9] N. Alonso-Alerca, P. Meessen, T. Ortín, Class. Quant. Grav. 17 (2000) 2783 .

[10] J. P. S. Lemos, Phys. Lett. B 353 (1995) 46.

[11] J. P. S. Lemos, V. T. Zanchin, Phys. Rev. D 54, (1996) 3840.

[12] J. P. S. Lemos, Class. Quantum Grav. 12, (1995) 1081.

[13] J. P. S. Lemos, "Rotating toroidal black holes in anti-de Sitter spacetimes and their properties", to be published in Proceedings of the Xth Portuguese Astronomy and Astrophysics Meeting, eds. J. P. S. Lemos, A. Mourão, L. Teodoro, R. Ugoccioni, (World Scientific, 2001); grqc/0011092.

[14] C. G. Huang, C-B. Liang, Phys. Lett. A 201 (1995) 27.

[15] D. Klemm, V. Moretti, L. Vanzo, Phys. Rev. D 57 (1998) 6127.

[16] J. P. S. Lemos, V. T. Zanchin, hep-th/9511188.

[17] J. P. S. Lemos, Phys. Rev. D 57 (1998) 4600.

[18] J. P. S. Lemos, Phys. Rev. D 59 (1999) 044020.

[19] S. G. Ghosh, "Charged Null Fluid Collapse in Anti-de Sitter Spacetimes", preprint (2000), Phys. Rev. D (to appear).

[20] C. Peça, J. P. S. Lemos, Phys. Rev. D 59 (1999) 124007.

[21] C. Peça, J. P. S. Lemos, J. Math. Physics 41 (2000) 4783.

[22] D. R. Brill, J. Louko, P. Peldán, Phys. Rev. D 65 (1997) 3600.

[23] L. Vanzo, Phys. Rev. D 56 (1997) 6475. 